\title{
Snapshot of anticoagulation practice in an inner London Borough in patients with known Atrial Fibrillation: Time for change
}

\author{
Dr Amna Burzic, Dr Maneesh Bhargava, Mrs Sarah Senganda \\ Stroke Department, North Middlesex University Hospital, London N18 1QX
}

\section{Introduction}

Atrial fibrillation $(\mathrm{AF})$ is the most common sustained cardiac arrhythmia. If untreated, it is a strong risk factor for embolic stroke.

The National Institute for Health and Care Excellence (NICE) guidelines for AF management emphasise the importance of stroke risk stratification and advise anticoagulation in people with a CHA2DS2VASc score of 2 or above, taking bleeding risk into account. However, many patients for whom anticoagulation is indicated do not receive appropriate therapy.

\section{Aims \& Objectives}

The aim of the audit was to review anticoagulation practice for stroke prevention in patients with $\mathrm{AF}$, based on recommendations by NICE.

\section{Objectives:}

- Identify patients presenting to the acute medical take from the community that are not receiving appropriate stroke prevention therapy, according to NICE guidelines

- Identify reasons why anticoagulation was not prescribed in this cohort

- Review appropriateness of anticoagulation in those receiving therapy

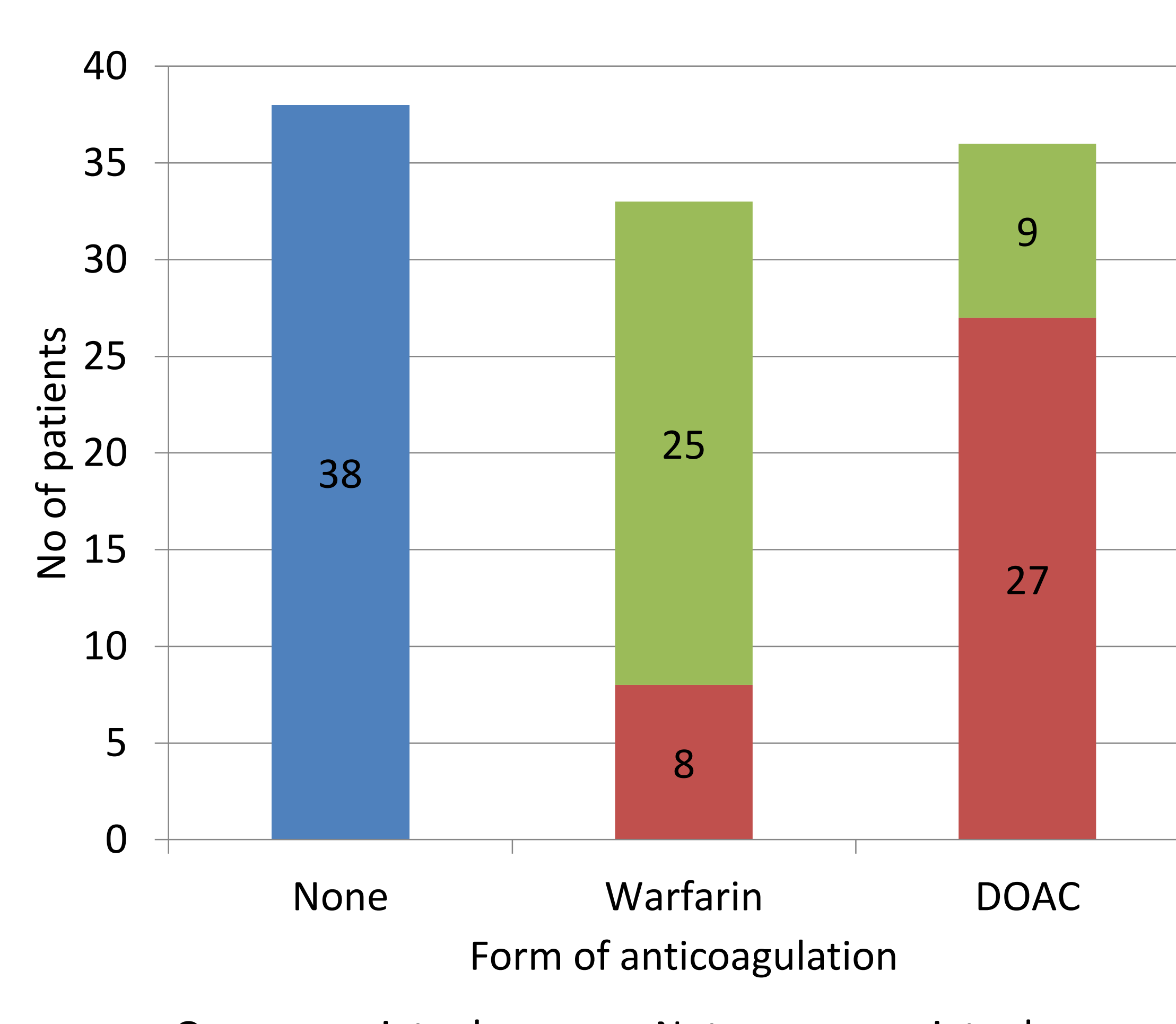

- On appropriate dose $\quad$ Not on appropriate dose

Chart 1. Number of patients not anticoagulated or anticoagulated with either warfarin or DOAC; including whether they were on appropriate dose.

Methods
All medical inpatients during the months of
November 2017 to February 2018 were
screened for a documented diagnosis of AF
prior to admission.
A standardized pro forma was designed to
collect information from inpatient notes and
electronic letters. In those with no hospital
documentation for withholding therapy,
general practitioners were contacted to
explore potential reasons further

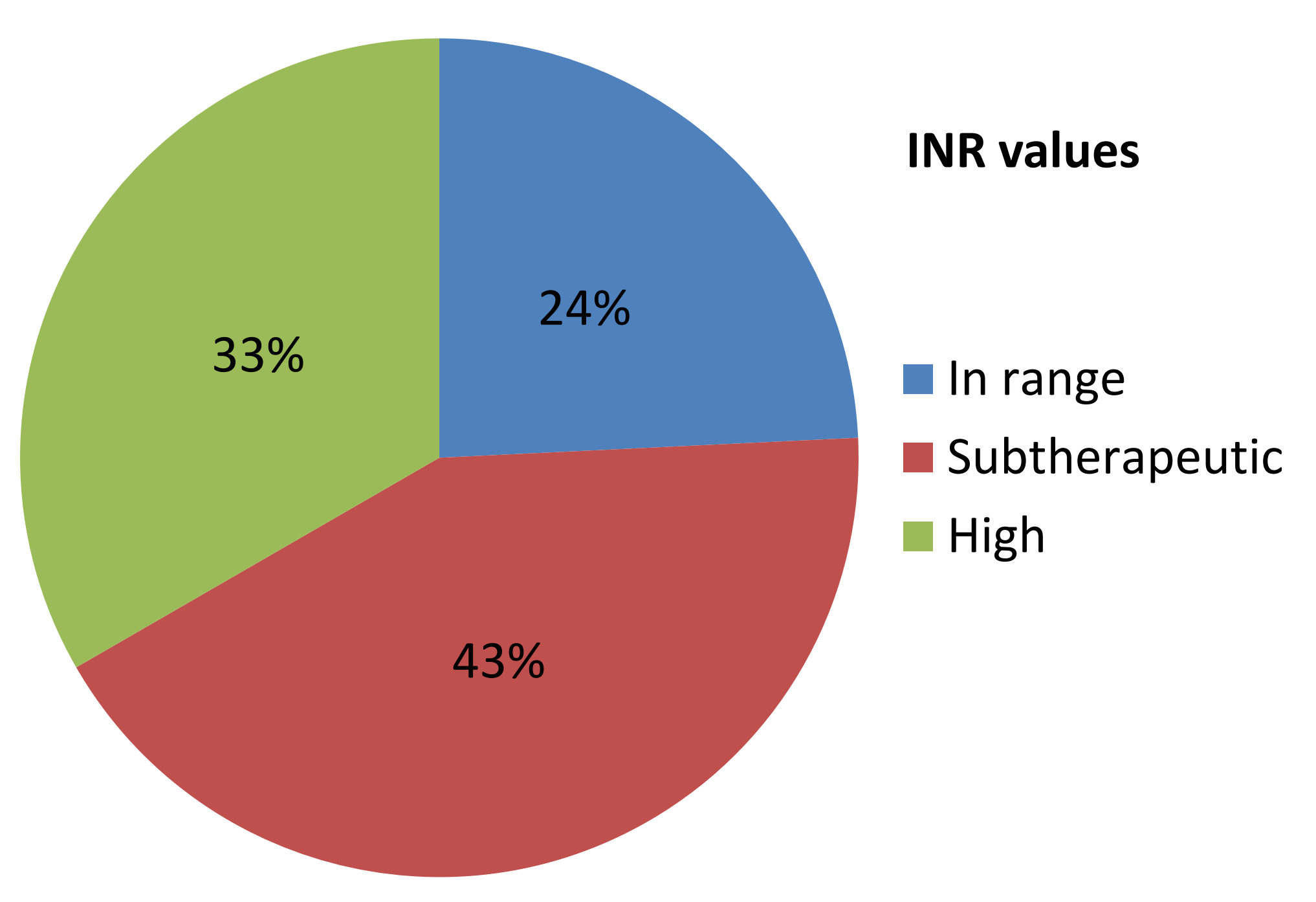

Chart 2. Number of patients anticoagulated with warfarin (total = 33) with an INR either in range, subtherapeutic or high on admission to hospital.

\section{Results}

107 patients in total were identified in the screening process.

$36 \%$ were not anticoagulated. Of these, only $37 \%$ had a documented reason for withholding therapy despite a mean CHA2DS2-VASc score of 5. Common reasons included bleeding risk and risk of falls.

Warfarin was the agent of choice in $48 \%$ of patients. Concerningly, $76 \%$ of patients on warfarin were admitted with an INR not in range; $56 \%$ of which were subtherapeutic.

Of the $52 \%$ on a DOAC, $25 \%$ were on an incorrect dose taking into account age (above 80 ), body-weight less than $61 \mathrm{~kg}$, or serum creatinine $133 \mathrm{micromol} /$ litre and over using the Cockcroft-Gault equation.

$37 \%$ of patients not anticoagulated prior to admission were initiated on therapy on discharge.

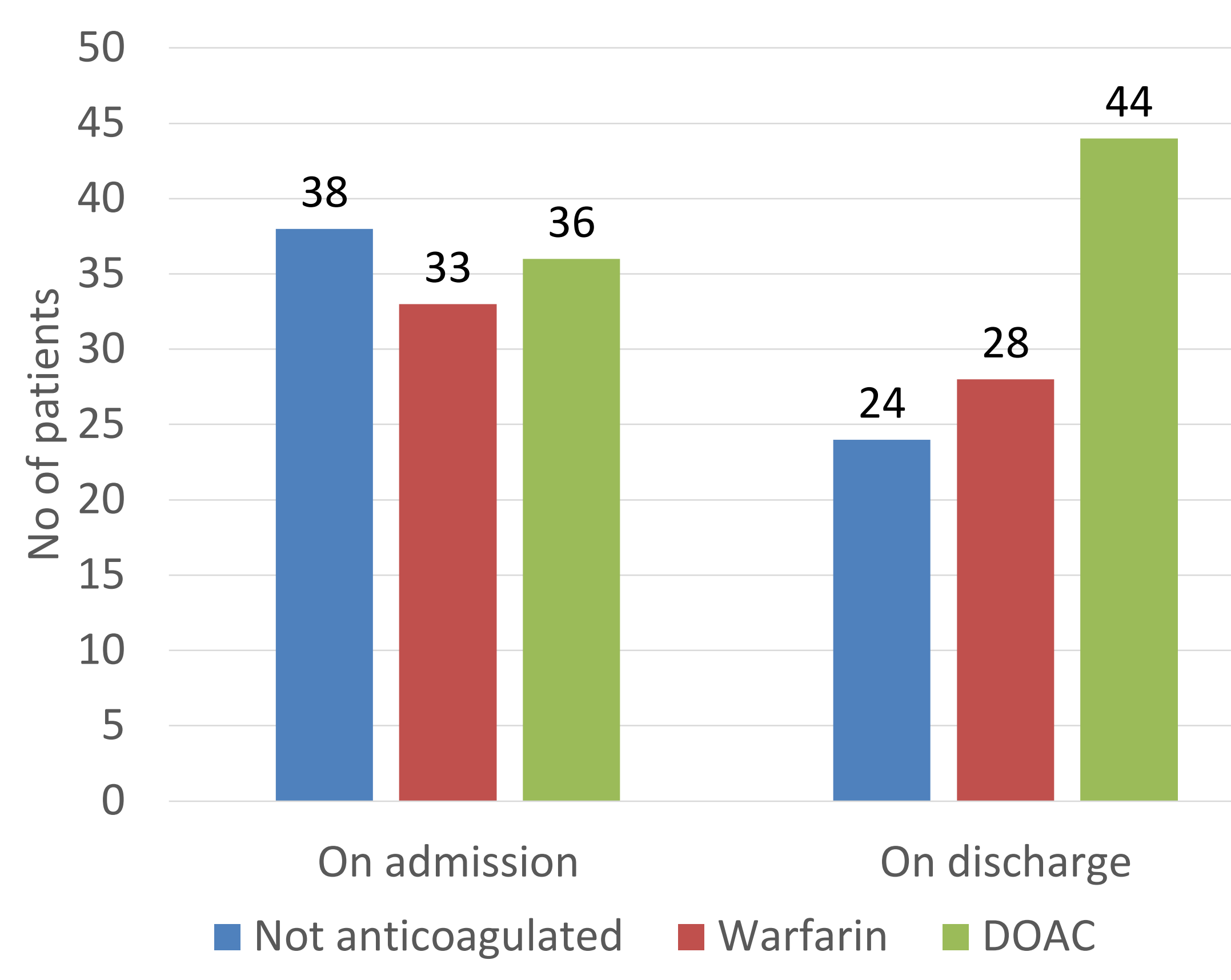

Chart 3. Number of patients with pre-existing AF managed consistently with NICE guidelines on stroke prevention on admission and discharge from a District General Hospital.

\section{Discussion}

Warfarin has been shown to be highly effective in AF-related stroke prevention but it's various shortcomings such as bleeding risk and numerous interactions with food and drugs render its use difficult. Because of this a large proportion of patients do not receive it or receive an inadequate dose as shown in our data.

Despite the development of DOACs, associated with a better safety profile and reduced need for monitoring, many practitioners remain reluctant to start anticoagulation or the reasons for their decisions may not always be clear in the medical notes, thus limiting our results.

Many patients are identified and initiated on therapy during hospital admissions. This suggests hospital practitioners were reassessing stroke risk during admission and modifying therapy appropriately.

\section{Conclusions}

Many patients in whom anticoagulation is indicated are receiving inappropriate dosage, or are not receiving treatment at all, thus increasing their risk of embolic stroke.

Clear guidance in the community on DOAC use in stroke prevention and when warfarin may be used in preference is of great importance. There is pressing need for regular review of both anticoagulation agent and dose, as well as more DOAC prescription in the community.

\section{Contact}

Dr Amna Burzic

North Middlesex University Hospital

Email:amna.burzic@nhs.net

\section{References}

Chugh S, Havmoeller R, Narayanan K, Singh D, Rienstra M et al. Worldwide Epidemiology of Atrial Fibrillation A Global Burden of Disease 2010 Study. Circulation. 2014;129(8):837-847

2. NICE. Atrial fibrillation: management. Clinical Guideline 180. NICE, 2014

3. Hanley C, Koewy P. Are the novel anticoagulants better than warfarin for patients with atrial fibrillation? Journal of Thoracic Disease. 2015;7(2):165-171 